\title{
Efficient Elastic Imaging of Single Atoms with Aberration-Corrected Scanning Transmission Electron Microscopy
}

\author{
Robert Hovden and David A. Muller \\ School of Applied \& Engineering Physics and Kavli Institute, Cornell University, Ithaca, NY 14853 \\ The fabrication of clean, mono-atomic-layer membranes such as graphene and boron nitride has greatly \\ simplified the imaging of individual light atoms in transmission electron microscopes (TEM) [1,2]. By \\ providing a supportive two-dimensional substrate of atoms, such membranes open doors to imaging \\ organic molecules - atom by atom. With a new generation of aberration-corrected microscopes, it is now \\ possible to image atoms with sub-angstrom detail. However, quantitative atomic imaging of biological \\ molecules on graphene membranes is still limited by the specimen's radiation damage [3]. In order to \\ overcome the limitations of specimen damage, microscopes require the most efficient collection of signal \\ possible. Increasing the signal per incident beam dose allows the exposure to be reduced and enables a \\ wider range of specimens to be imaged in a shorter time.
}

Here we characterize the detection efficiency and interpretability of imaging single atoms by elastic electron scattering over a range of detector geometries in a scanning transmission electron microscope (STEM). STEM microscopes can also convey atomic-resolution chemical information from directlyinterpretable scattered intensities [4]. Typically, in order to ensure a directly interpretable signal over a range of amorphous and crystalline specimens, a high angle annular dark field (HAADF) detector with a sufficiently-large inner angle is used [5]. However, we show for the special case of well-resolved, atomically-thin specimens, such requirements for interpretability are too conservative. In the case of isolated single atoms, the signal remains monatomic with atomic number even for inner angles just outside the collection angle - a regime close to that proposed by Crewe [4].

As elucidated by Fig. 2, when the inner detector angle is reduced to just outside the convergence angle the carbon signal is increased by a factor of 6.0 and 2.79 over HAADF (80-240mrad) and MAADF (55$240 \mathrm{mrad}$ ) detector geometries respectively. Fig 1 shows a two-dimensional map of a single carbon atom imaged over the entire detector parameter space. One interesting regime, annular bright field (ABF), has been shown to provide useful imaging conditions for light atom detection in thicker samples[6], however the overlap with the unscattered beam (Fig. 1a) lowers the SNR (Fig. 1b) thus increasing the dose requirements for imaging. From Fig. $1 \mathrm{~b}$ and Fig 2 we see the optimal low-dose imaging of single atoms occurs in the low-angle (LAADF) regime. Although LAADF provides an appreciable increase of signal, imaging of light atoms with $1 \AA$ resolution will still require doses larger than the $0.1 \mathrm{C} / \mathrm{cm} 2$ damage threshold typical to polymeric molecules. LAADF simulations of the functionalized nucleotide, Adenine Triphosphate (ATP), indicate an ideal dose requirements greater than $100 \mathrm{C} / \mathrm{cm} 2$ (Fig. 3a) - much larger than the $\sim 0.2 \mathrm{C} / \mathrm{cm}^{2}$ dose limit reported by Isaacson [7]. Thus, structural imaging by an annular detector will only be possible for more stable compounds, or those containing heavier elements.

[1] J.C. Meyer, A. Zettl, et. al., Nano Letters, vol. 8 p.3582 (2008)

[2] O.L. Krivanek, et. al., Nature, vol. 464, p. 571 (2010)

[3] M. Ohtsuki, M.S. Isaacson, and A.V. Crewe, Proc.Nat. Acad. Sciences USA, vol. 76, p.1228 (1979)

[4] A.V. Crewe, J. Wall, and J. Langmore Science, vol. 168, pp.1338 (1970)

[5] H. Rose and J. Fertig Ultramicroscopy, vol. 2, p. 77 (1976)

[6] S.D. Findlay, et. al., APL, vol. 95 p. 191913 (2009)

[7] M.S. Isaacson, Journal of Chemical Physics, vol. 56, p.1813 (1972)

[8] Funded by the Semiconductor Research Corporation and CNS (NSF \#EEC-0117770, 0646547) 

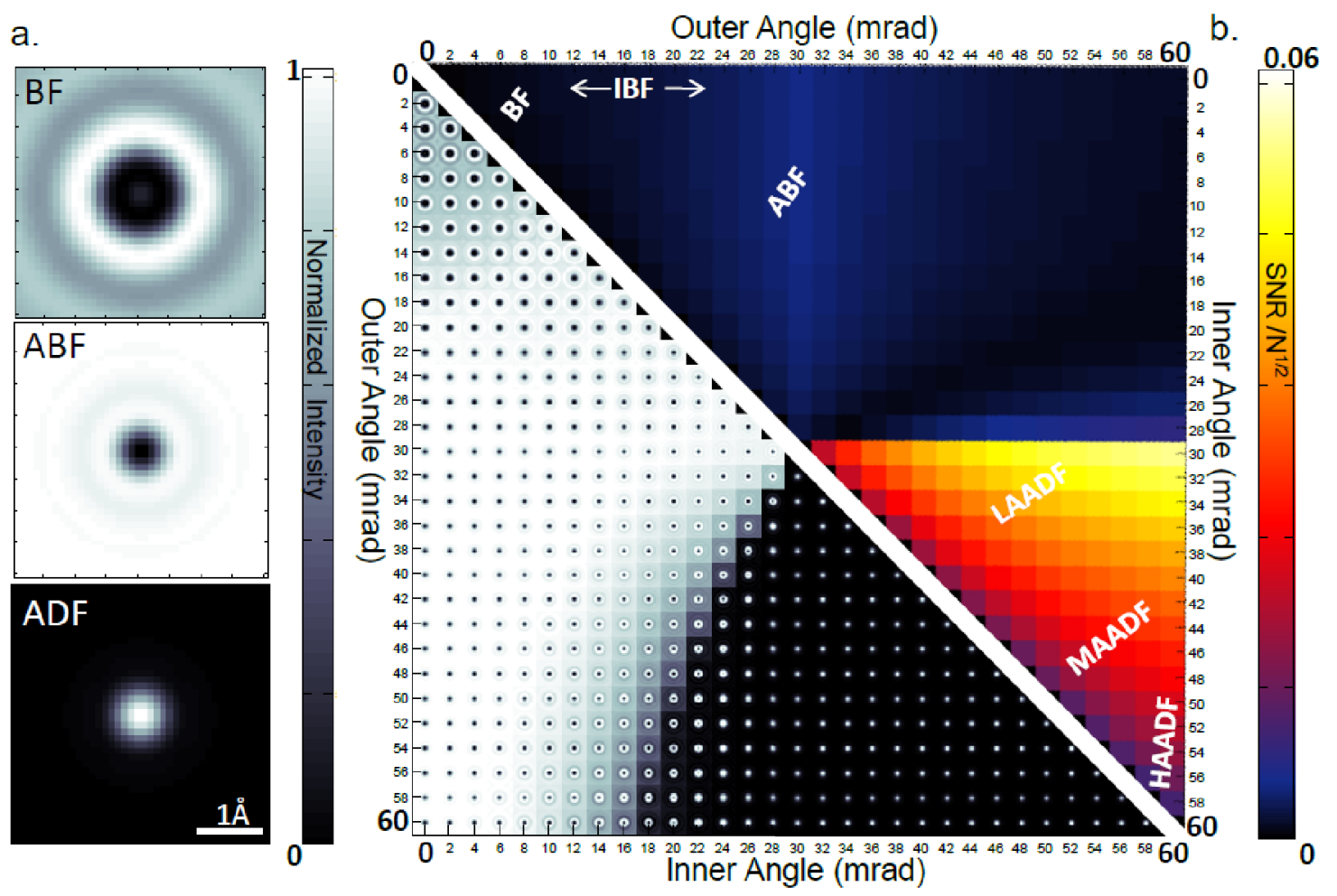

FIG 1. a.) Carbon atom as imaged by three popular detector regimes, bright field (BF), annular bright field (ABF), and annular dark field (ADF), in a STEM are shown left. Calculated by $100 \mathrm{keV}$ multislice simulation. A map of comprised of carbon atoms imaged over a range of inner and outer detector angles is also shown. Each subimage ( $4 \AA$ wide) containing an atom has been normalized to its own max value. b.) The corresponding signal to noise coefficient due to shot noise, $\mathrm{SNR} / \mathrm{N}^{1 / 2}$ where $\mathrm{N}$ is the number of incident electrons per pixel, is shown in a mirrored fashion to the map in (a.). Note that the SNR increases as one goes from a high to a low angle annular dark field (LAADF to HAADF) detector.

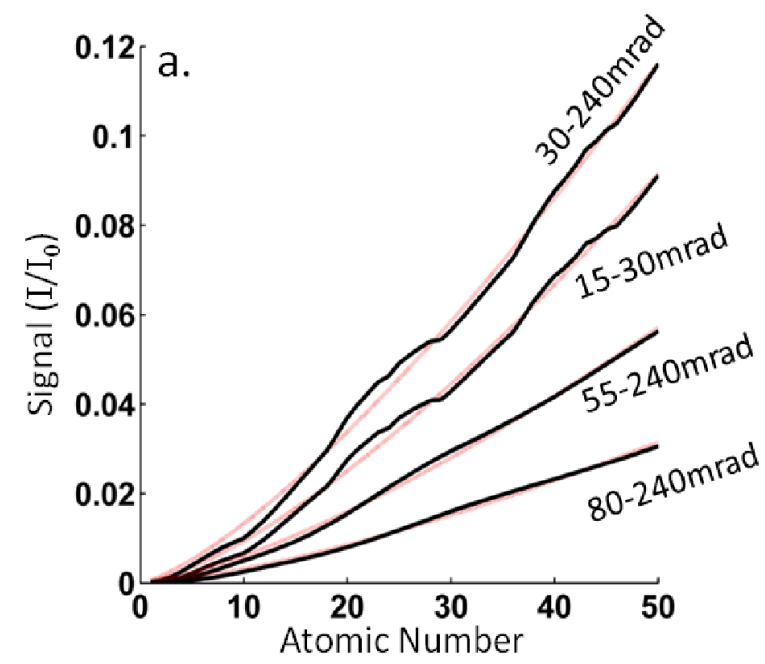

FIG 2. Simulated signal of a scattered $60 \mathrm{keV}$, $30 \mathrm{mrad}$ convergent electron beam scattered off of single atoms for various detector geometries. Signals monotonically increase as a power of atomic number.
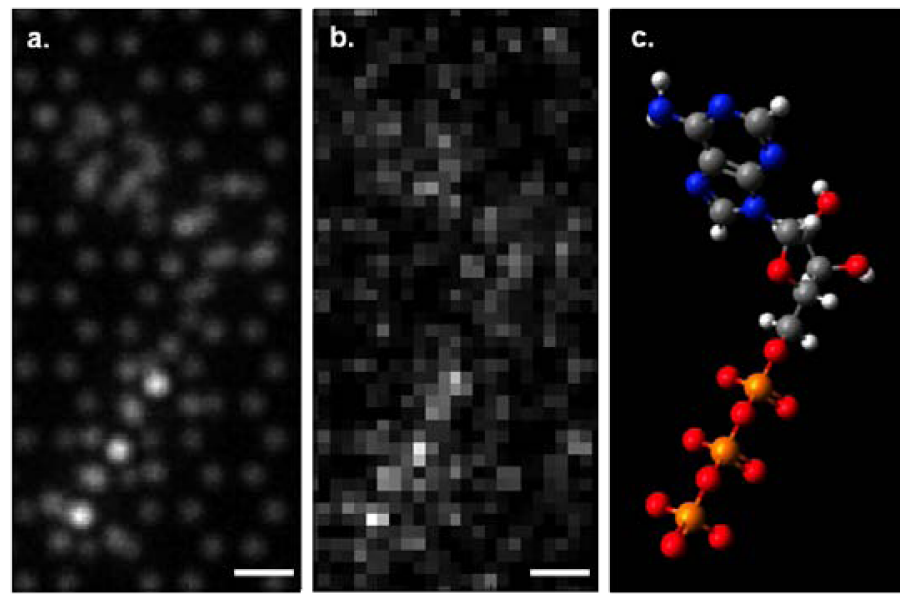

FIG 3. Multislice simulation $(60 \mathrm{keV}, \alpha=30$ mrad) of ATP imaged on graphene substrate using LAADF (30-250mrad) and beam dose a) $250 \mathrm{C} / \mathrm{cm}^{2}$ and b) $5.0 \mathrm{C} / \mathrm{cm}^{2}$. c) toy model description of ATP: P-orange, O-red, H-white, C-grey, N-blue 